\title{
Cross layer scheduling algorithm for LTE Downlink
}

\author{
Popovska Avramova, Andrijana; Yan, Ying; Dittmann, Lars
}

Published in:

2012 20th Telecommunications Forum (TELFOR)

Link to article, DOI:

10.1109/TELFOR.2012.6419208

Publication date:

2012

Link back to DTU Orbit

Citation (APA):

Popovska Avramova, A., Yan, Y., \& Dittmann, L. (2012). Cross layer scheduling algorithm for LTE Downlink. In 2012 20th Telecommunications Forum (TELFOR) (pp. 307-310). IEEE.

https://doi.org/10.1109/TELFOR.2012.6419208

\section{General rights}

Copyright and moral rights for the publications made accessible in the public portal are retained by the authors and/or other copyright owners and it is a condition of accessing publications that users recognise and abide by the legal requirements associated with these rights.

- Users may download and print one copy of any publication from the public portal for the purpose of private study or research.

- You may not further distribute the material or use it for any profit-making activity or commercial gain

- You may freely distribute the URL identifying the publication in the public portal

If you believe that this document breaches copyright please contact us providing details, and we will remove access to the work immediately and investigate your claim. 


\title{
Cross layer scheduling algorithm for LTE Downlink
}

\author{
Andrijana Popovska Avramova, Ying Yan, and Lars Dittmann
}

\begin{abstract}
The LTE standards is leading standard in the wireless broadband market. In order to accommodate the increased demand for mobile data services the radio interface in the LTE standard is enhanced with advanced technologies such as OFDMA (Orthogonal Frequency Division Multiple Access) and MIMO (multiple antenna) techniques. The Radio Resource Management at the base station plays major role in satisfying the users demand for high data rates and QoS. Resources for both downlink and uplink transmission need to be assigned such that the capacity, throughput, and cell edge performance are optimized. This paper evaluates a cross layer scheduling algorithm that aims at minimizing the resource utilization. The algorithm makes decisions regarding the channel conditions and the size of transmission buffers and different QoS demands. The simulation results show that the new algorithm improves the resource utilization as well as provides better guaranties for QoS.

Keywords - LTE, buffer-aware, subcarrier allocation, QoS.
\end{abstract}

\section{INTRODUCTION}

$\mathrm{N}$ ot only because of the technology but also because it fulfills defined requirements for a pure $4 \mathrm{G}$ generation and backward compatibility with its previous generations (GSM, UMTS...), 3GPP Long Term Evolution (LTE) is leading the wireless mobile world [1]. The air interface of this all-IP-based network architecture utilizes the SCFDMA in the uplink and OFDMA in the downlink enhanced by multiple antenna systems, supporting both the time (TDD) and frequency (FDD) division duplex modes. Due to the all-IP based architecture, the air interface needs to accommodate a mixture of real and non-real time services. This means that the all traffic including delay sensitive services needs to be scheduled [2].

The main purpose of the LTE scheduling system in the Base Station (BS) is to prioritize and allocate the available frequency-time resources to specific single user equipment (UE). The scheduling is at the MAC (Medium Access Control) layer, it is not standardized and it is an

First A. Popovska Avramova is with the Department of Photonics Engineering, Technical University of Denmark, Ørsteds Plads, Building 343, Office 126, 2800 Kgs. Lyngby, Denmark (phone: 004545253852; e-mail: apop@fotonik.dtu.dk).

Corresponding Second Ying Yan, is now with the Department of Photonics Engineering, Technical University of Denmark, Ørsteds Plads, Building 343, Office 126, 2800 Kgs. Lyngby, Denmark (phone: 004545253613; e-mail: yiya@fotonik.dtu.dk).

Third L. Dittmann is with the Department of Photonics Engineering, Technical University of Denmark, Ørsteds Plads, Building 343, Office 126, 2800 Kgs. Lyngby, Denmark (phone: 004545253851; e-mail: ladit@fotonik.dtu.dk). implementation specific mechanism. The overall system performance and reusability of the resources are mainly influenced by the scheduling algorithm [4]. The design of a downlink scheduling algorithm is a complex procedure and presents a number of design challenges, such as maximization of system capacity and spectral efficiency, bit error performances, fairness approach, etc.

So far, there has been a lot of research in modeling the scheduler in order to achieve the highest performance while avoiding latency and starvation problems. In this paper we evaluate a cross layer scheduler scheme that considers the physical layer (channel conditions), application layer (service type) and RLC layer (transmission buffer size). The results are compared with the maximum capacity algorithm with QoS service classification. The algorithms are evaluated on different criteria, such as achieved air interface throughput and delay for each type of service.

This paper is organized as follows. In section II, we give some general insights on scheduling algorithms present in the literature. Section III describes the packet scheduler model and the proposed scheduling algorithm. Section IV presents the simulation parameters, while the Section $\mathrm{V}$ provides a discussion of the simulation results. Concluding remarks are offered in the last section.

\section{OVERVIEW OF PACKET SCHEDULING SCHEMES}

This section offers a brief overview of packet scheduling algorithms present in the literature based on [1]-[12]. The packet scheduling is an optimization problem where the scheduling function can be constructed such as to define the way the objectives of the optimization are reached. In accordance with the manner the optimization is achieved, a simple classification of this function can be done: rate-adaptive, delay adaptive and utility based schemes.

The rate adaptive process can be instantaneous, proportional-fair and rate adaptive with margin. The instantaneous (maximum capacity) algorithm exploits multiuser diversity by assigning the subcarriers to those users that experience the best channel conditions. This algorithm tends to maximize the overall system capacity but fails to ensure fairness or any QoS guarantee. Additionally this algorithm does not improve cell edge performance and it can lead to starvation for users that have bad channel conditions. The proportional fair algorithm provides fairness among users such that the minimum data rate enforced on each user is maximized. This algorithm tends to achieve maximum capacity with constraints of data rate fairness to all users. The rate 
adaptive with margin additionally considers QoS requirements such that it tends to guarantee minimum (threshold) data rate to each user.

The delay based algorithms tend to optimize the overall system delay under certain constraints. The delay based function can be instantaneous where the users that would experience minimum delay are prioritized. It can also be proportional where the maximum delay enforced on each user is minimized. The rate adaptive and delay adaptive can be considered in combination. Additionally the objective function can involve certain degree for cell edge performance, and/or cell load balancing.

Utility theory can be applied in the design of the packet scheduler. The utility function can be derived from surveys, user's profiles and behavior, differentiation between concurrent services executing at one user, overall network traffic.

\section{PACKET SCHEDULER DESIGN}

\section{A. General Scheduler Design}

The efficient utilization of the air interface in a scheduling system as LTE, is achieved as a combination of subcarrier allocation, adaptive modulation and coding and power allotment. Due to the limited radio resources such as available spectrum, power and equipment utilities (for ex. number of antennas), the resource allocation needs to be done such that these resources are utilized in most optimal way while the system performances are maximized. In order to simplify the resource management, the decision process can be divided into two components [5][9]:

Time Domain: The goal is to generate a prioritized list of UEs (their radio bearers). It can be based on different parameters such as HARQ retransmission, QoS requirements, users' profiles etc. The number of users that can be scheduled at each TTI is limited by the size of the PDCCH (physical downlink control channel).

Frequency domain, subcarrier allocation: Here the goal is to decide on the ordering and amount of the resources (subcarriers) assigned to each user. Usually it is based on the channel quality indicators, but can also include the QoS requirements, (transmit and/or receive) buffer status etc. The size of the PDSCH and PUSCH (Physical Downlink/Uplink Share Channel) limits the total number of resource elements assigned.

Frequency domain, bit selection (or power allocation): The power assigned to each subcarrier defines the amount of bits to be transmitted. Total transmission power and cell interference govern the power assignment.

\section{B. Proposed Scheduler Design}

This section presents the proposed scheduling algorithm based on the channel quality indicator and the transmission buffer size (TX CAP). In the time domain the users (or radio bearers) are sorted according to the service priority. The VoIP service has the highest priority, then the Video streaming service followed by the Web browsing service and the FTP service at last. In the frequency domain, the resource block $(\mathrm{RB})$ assignment is done according to the transmission buffer size. The users with the same service priority are sorted according the transmission buffer size and the channel quality indicators. The users with the larger buffer size are assigned more RBs. Additionally if the data that is available for transmission does not fill more than one resource block, the users is not considered for scheduling. Regarding the power allocation, equal power is assumed for each subcarrier.

In order to confirm the performance improvement of the proposed scheduler, the results are compared with the maximum capacity algorithm where the UE are again sorted according the channel quality indicators (referred to as MAX CAP algorithm).

\section{Simulation Parameters}

\section{A. Traffic Parameters}

Four different service types have been considered: VoIP, Video service, Web browsing and FTP sessions. The purpose for choosing mixed data traffic is to show the scheduler design impact on the QoS and different services. Based on the traffic parameters for different services in [15][16] and [17], the traffic parameters for the four services considered during the simulations are shown in Table 1 .

TABle 1: Traffic Source Parameters

\begin{tabular}{|c|c|c|}
\hline & Parameter & Assumption \\
\hline $\begin{array}{l}\text { 응 } \\
>\end{array}$ & $\begin{array}{l}\text { Silence length } \\
\text { Talk length } \\
\text { Call Duration } \\
\text { Inter-call duration } \\
\text { Encoding scheme }\end{array}$ & $\begin{array}{l}\text { Exponential }(0.65 \mathrm{sec}) \\
\text { Exponential }(0.352 \mathrm{sec}) \\
\text { Constant }(90 \mathrm{sec}) \\
\text { Exponential }(30 \mathrm{sec}) \\
\text { GMS EFR }\end{array}$ \\
\hline $\begin{array}{l}\stackrel{8}{0} \\
\stackrel{8}{>} \\
>\end{array}$ & $\begin{array}{l}\text { Inter-frame time } \\
\text { Nr. pkts per frame } \\
\text { Packet size } \\
\text { (bytes) }\end{array}$ & $\begin{array}{l}\text { Constant }(0.1 \mathrm{sec}) \\
\text { Constant }(8 \mathrm{pkts}) \\
\text { Truncated Pareto } \\
(\text { Mean }=100, \mathrm{Max}=250) \\
\text { location }=40 \text { shape }=1.2\end{array}$ \\
\hline & $\begin{array}{l}\text { Main Object Size } \\
\text { (bytes) } \\
\text { Embedded Object } \\
\text { Size (bytes) } \\
\text { Nr. embedded } \\
\text { objects per page } \\
\text { (rand nr - } \\
\text { location) } \\
\text { Reading Duration }\end{array}$ & $\begin{array}{l}\text { Truncated Lognormal min } \\
=100, \text { max }=2 \mathrm{Mb}(\text { mean } \\
=10710, \text { std. Dev. } 25032) \\
\text { Truncated Lognormal min } \\
=50, \text { max }=2 \mathrm{Mb}(\text { mean }= \\
7758, \text { std. Dev. } 126168) \\
\text { Truncated Pareto } \\
(\text { shape }=1.1, \text { location }=2) \\
\text { max }=53 \\
\text { Exponential }(30 \mathrm{sec} .)\end{array}$ \\
\hline 芫 & $\begin{array}{l}\text { File size (Mbytes) } \\
\text { Reading time }\end{array}$ & $\begin{array}{l}\text { Truncated Lognormal } \\
\max =5(\text { mean }=2, \\
\text { Std. Dev. }=0.722) \\
\text { Exponential }(180 \mathrm{sec})\end{array}$ \\
\hline
\end{tabular}

\section{B. Simulation Tool}

OPNET Modeler Tool has been used in order to perform evaluation of the proposed scheduling scheme. Built-in LTE models were used in order to ensure 
confidence and correctness of the evaluation.

Current OPNET version (16) does not support adaptive modulation and coding in LTE. Therefore the model was enhanced with this feature by using the Effective Exponential SINR Mapping as described in [19].

\section{Network Simulation Parameters}

The simulations consider a single-cell multi-user scenario. A scenario of $16 \mathrm{UEs}$, such that there are 4 UEs for each service class, and average distance to the eNB of 450 meters is considered.

The simulations are performed for minimum specified bandwidth for LTE and the ITU "Pedestrian A" channel model. It is assumed that a fixed $80 \%$ of the PDCCH is to be used for downlink assignments. Table 2 shows the rest of the system configuration parameters.

Table 2: Network Simulation Parameters

\begin{tabular}{l|l}
\hline Parameter & Value \\
\hline System Bandwidth & $1.4 \mathrm{MHz}$ \\
Multiplexing & FDD \\
Subcarrier Spacing & $15 \mathrm{kHz}$ \\
Cycle Prefix & Normal \\
Number of eNB & 1 (no Sectors) \\
Number of UE & 16 users \\
Channel Profile & ITU Pedestrian A \\
RLC mode & UN-ACK Mode \\
CQI measurements & Wideband \\
Time window for CQI & $1 \mathrm{~ms}$ \\
Reporting interval & $5 \mathrm{~ms}$ \\
Simulation Duration & $1700 \mathrm{sec}$ \\
\hline
\end{tabular}

\section{Simulation Results}

This section presents the results from the simulation based analysis for the scheduling scheme and configuration presented in the previous sections. The comparison metrics that are presented are the average achieved throughput and delay for each class of service. Several simulations have been executed for different seed values and the results have been averaged.

The simulations have shown that utilization of the $\mathrm{PDCCH}$ has been reduced with the new scheduling scheme (TX CAP). The average utilization with the TX CAP scheme is $85 \%$ compared to $100 \%$ with the MAX CAP scheme. This is result from the fact that UEs with larger buffers are prioritized, and therefore the assigned higher numbers of RB per UE is higher. This leads to lowered number of UEs scheduled in one TTI as all RB are already allocated. The available space in the PDCCH can be further utilized for downlink data transmission and therefore the overall throughput can be increased.

Fig. 1 represents the average end-to-end delay for delay sensitive services (VoIP and Video Streaming), page response time for HTTP service and file download time for FTP service. As it can be seen from the figure, the delay sensitive services have reduced average packet endto-end delay with the TX CAP algorithm. The reason for this is that due to the QoS classification these services will have higher priority and they will be always considered for transmission in first place. On the other hand the HTTP service and FTP service are slightly degraded. The average page download time is increased by $0.5 \mathrm{sec}$, while the average file download time is increased by $7 \mathrm{sec}$.



Fig. 1. Average delay for different services

Fig. 2 represents the average throughput measured at different UEs and averaged for each service type. Only the VoIP service has certain degradation in the throughput compared to the other services. All other services have increased throughput, especially the FTP services. The reason for this is that when HTTP and FTP users are scheduled, the number of UEs scheduled for that TTI is lowered as FTP users will be allocated larger number of RB. Here the rest of the PDCCH not used for assignments is utilized for downlink data transmissions.

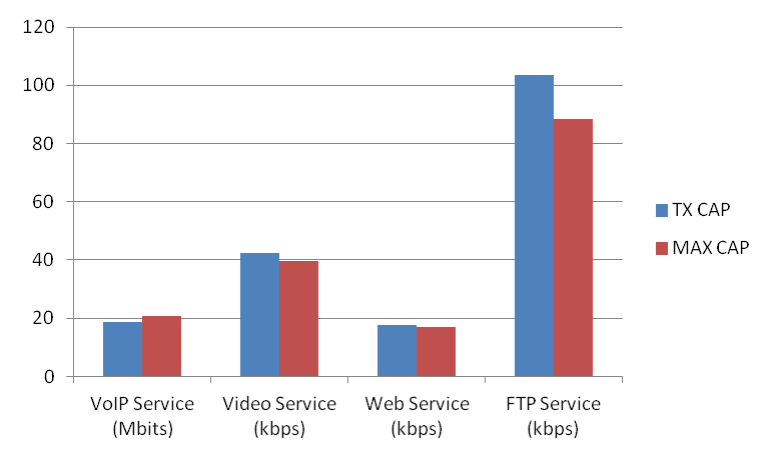

Fig. 2. Average throughput for different services

\section{CONCLUSION}

This paper elaborates on the LTE downlink scheduling schemes and gives an evaluation by simulation with builtin LTE OPNET model. The results of the proposed scheme have been compared to the MAX CQI algorithm with QoS awareness.

The results have shown that when transmission buffer size is considered by the scheduler in combination with channel conditions and QoS classification, the delay requirements for real time services can be improved. Additionally the number of colons required by the PDCCH can be reduced and used for downlink transmission. Therefore the data rate for non-real time services can be improved.

In this phase of the project, only wideband measurements have been considered. In the future, we plan to include sub-band measurements. Additionally the time 
domain scheduling in case of increased number of VoIP users can lead to degraded performance of other service types, especially the FTP services. Therefore other parameters need to be further considered in order to satisfy the minimum QoS demands for non-real type of traffic.

\section{REFERENCES}

[1] Popovska Avramova, Yan, Y.; Dittmann, L.; "Modeling and Simulation of Downlink Subcarrier Allocation Schemes in LTE", Proceedings of OPNETWORK2012, 2012, OPNET, Presented at: OPNETWORK, Washington DC, 13-17 August 2012

[2] Gavrilovska, L.; Talevski, D.; , "Novel scheduling algorithms for LTE downlink transmission," Telecommunications Forum (TELFOR), 2011 19th , vol., no., pp.398-401, 22-24 Nov. 2011

[3] Pandharipande, A.; Kountouris, M.; Ho Yang; Hyoungwoon Park; , "Subcarrier allocation schemes for multiuser OFDM systems," Signal Processing and Communications, 2004. SPCOM '04. 2004 International Conference on , vol., no., pp. 540- 544, 11-14 Dec. 2004

[4] Shih-Jung Wu; Liou Chu; , "A Novel Packet Scheduling Scheme for Downlink LTE System," Intelligent Information Hiding and Multimedia Signal Processing (IIH-MSP), 2011 Seventh International Conference on , vol., no., pp.25-28, 14-16 Oct. 2011

[5] Zaki, Y.; Weerawardane, T.; Gorg, C.; Timm-Giel, A.; , "MultiQoS-Aware Fair Scheduling for LTE," Vehicular Technology Conference (VTC Spring), 2011 IEEE 73rd , vol., no., pp.1-5, 15-18 May 2011

[6] Kela, P.; Puttonen, J.; Kolehmainen, N.; Ristaniemi, T.; Henttonen, T.; Moisio, M.; , "Dynamic packet scheduling performance in UTRA Long Term Evolution downlink," Wireless Pervasive Computing, 2008. ISWPC 2008. 3rd International Symposium on, vol., no., pp.308-313, 7-9 May 2008

[7] Sadiq B.; Madan R.; Sampath A.; , "Downlink Scheduling for Multiclass Trafic in LTE", Hindawi Publishing Corporation, EURASIP Journal on Wireless Communications and Networking, Volume 2009, Article ID 510617, March 2009

[8] Hosein, P.; , "Resource Allocation for the LTE Physical Downlink Control Channel," GLOBECOM Workshops, 2009 IEEE , vol., no., pp.1-5, Nov. 30 2009-Dec. 42009

[9] M. Salah, N.A. Ali, A.E.M. Taha and H.S. Hassanein, "Designing Standard-Compliant LTE Schedulers", ; Wireless World Research Forum, Doha, Qatar, April 2011.

[10] Peng Cheng; Guanding Yu; Zhaoyang Zhang; Peiliang Qiu; , "A Cross-Layer Fair Resource Allocation Algorithm for OFDMA Systems," Communications, Circuits and Systems Proceedings, 2006 International Conference on , vol.2, no., pp.1342-1346, 25-28 June 2006

[11] Salman, M.I.; Chee Kyun Ng; Noordin, N.K.; Mohd Ali, B.; Sali, A.; , "Energy efficient transmission for LTE cellular system," Computer and Communication Engineering (ICCCE), 2012 International Conference on, vol., no., pp.222-227, 3-5 July 2012

[12] Jinri Huang; Zhisheng Niu; , "Buffer-Aware and Traffic-Dependent Packet Scheduling in Wireless OFDM Networks," Wireless Communications and Networking Conference, 2007.WCNC 2007. IEEE , vol., no., pp.1554-1558, 11-15 March 2007

[13] Yan Lin; Guangxin Yue; , "Channel-Adapted and Buffer-Aware Packet Scheduling in LTE Wireless Communication System," Wireless Communications, Networking and Mobile Computing, 2008. WiCOM '08. 4th International Conference on, vol., no., pp.14, $12-14$ Oct. 2008

[14] Ameigeiras, P.; Wang, Y.; Ortiz, J. N.; Mogensen, P. E.; LopezSoler, J. M.; , "Traffic models impact on OFDMA scheduling design", EURASIP Journal on Wireless Communications and Networking 2012, Feb 2012.

[15] H. Wang and V. B. Iversen, "Hierarchical downlink resource management framework for OFDMA based WiMAX systems," in Wireless Communications and Networking Conference, 2008. WCNC 2008. IEEE, 2008, pp. 1709-1715

[16] 3GPP TR 25.892 V6.0.0 (2004-06), Technical Report 3rd Generation Partnership Project; Technical Specification Group Radio Access Network; Feasibility Study for Orthogonal Frequency Division Multiplexing (OFDM) for UTRAN enhancement

[17] Yong Fan; Kuusela, M.; Lunden, P.; Valkama, M.; , "Downlink VoIP Support for Evolved UTRA," Wireless Communications and Networking Conference, 2008. WCNC 2008. IEEE , vol., no., pp.1933-1938, March 31 2008-April 32008
[18] Sandanalakshmi, R.; Palanivelu, T.G.; Manivannan, K.; , "Effective SNR mapping for link error prediction in OFDM based systems," Information and Communication Technology in Electrical Sciences (ICTES 2007), 2007. ICTES. IET-UK International Conference on , vol., no., pp.684-687, 20-22 Dec. 2007

[19] C. Mehlfuhrer, M. Wrulich, J.C Ikuno, D.Bosanska, and M. Rupp, "Simulating the long term evolution physical layer, " in Proc. of the 17th European Signal processing conference (EUSIPCO 2009), Glasgow, Scotland, Aug. 2009.

[20] 3GPP TS 36.213 V10.4.0 (2011-12), 3rd Generation Partnership Project; Technical Specification Group Radio Access Network; Evolved Universal Terrestrial Radio Access (E-UTRA); Physical layer procedures 